\title{
TIME SERIES MODELING OF SYSTEM SELF-ASSESSMENT OF SURVIVAL
}

\author{
Huitian Lu and Willian J. Kolarik \\ Department of Industrial Engineering \\ Texas Tech University \\ Lubbock, TX 79409-3061 \\ Phone: (806) 742-3543 \\ E-mail: wkolarik@coe3.coe.ttu.edu
}

\section{RECEIVED \\ MAY 241999 \\ OSTI}

\begin{abstract}
Self-assessment of survival for a system, subsystem or component is implemented by assessing conditional performance reliability in real-time, which includes modeling and analysis of physical performance data. This paper proposes a time series analysis approach to system self-assessment (prediction) of survival. In the approach, physical performance data are modeled in a time series. The performance forecast is based on the model developed and is converted to the reliability of system survival. In contrast to a standard regression model, a time series model, using on-line data, is suitable for the real-time performance prediction. This paper illustrates an example of time series modeling and survival assessment, regarding an excessive tool edge wear failure mode for a twist drill operation.
\end{abstract}

Key words: Performance reliability, Reliability prediction, Time series, Survival assessment

\section{INTRODUCTION}

System reliability is traditionally estimated by analyzing life test data. In reliability engineering, it is commonly accepted that the reliability characteristic of a system can be quantitatively modeled as a reliability function. This approach considers only two possible states of an operating system: a state of functioning and a state of failure. In this way, the system under consideration is accepted as a 'black box' which performs the required function until it fails. However, customers as well as designers and engineers are interested in sustained system performance over time, and prefer to proact before system failure happens. They desire to maximize product performance as well as minimize costs, all in real-time. This desire leads to a need for real-time conditional reliability estimates; so that the information of the system performance and failure can be factored into operating and maintenance related decisions. Here, real-time physical data is mined for degradation clues, which are in turn transformed to conditional probabilities of survival or reliability.

A quantitative model, driven by on-line data, is necessary to capture the essence of the conditional reliability related to system performance. In general, given performance measure $y(t)$ and its critical limit value $y_{C L}$ for a defined failure mode, the model predicts the conditional performance reliability, or $R(t+\Delta t \mid t)$, at current time $t$, based upon the observations of $y(t)$. Performance measure modeling and conditional reliability prediction have been studied by Kim (1991), Kim and Kolarik (1992), and Chinnam et al. (1996) using regression models and neural network models. The purpose of this paper is to report on a similar study based on time series modeling. Usually a future value of a time series is relative to its past values and driven by a white noise process. The task in survival assessment in time series modeling is to model system performance measures in a time series and convert the model forecast into a conditional performance reliability.

In this paper, on-line drill thrust force data, collected from a steel twist drill operation, are used to demonstrate performance prediction regarding a drill failure mode of excessive wear. Section 2 briefly discusses the concept of survival assessment based on physical performance monitoring. Section 3 discusses the time series approach to modeling performance data and extracting conditional reliability predictions. The 


\section{DISCLAIMER}

This report was prepared as an account of work sponsored by an agency of the United States Government. Neither the United States Government nor any agency thereof, nor any of their employees, make any warranty, express or implied, or assumes any legal liability or responsibility for the accuracy, completeness, or usefulness of any information, apparatus, product, or process disclosed, or represents that its use would not infringe privately owned rights. Reference herein to any specific commercial product, process, or service by trade name, trademark, manufacturer, or otherwise does not necessarily constitute or imply its endorsement, recommendation, or favoring by the United States Government or any agency thereof. The views and opinions of authors expressed herein do not necessarily state or reflect those of the United States Government or any agency thereof. 


\section{DISCLAIMER}

Portions of this document may be illegible in electronic image products. Images are produced from the best available original document. 
application to tool performance survival assessment in the drilling operation is presented in Section 4.

\section{ASSESSMENT OF SURVIVAL}

System survival assessment is implemented by modeling and predicting system performance variable $y(t)$ across time, $t=0,1,2, \ldots$ (a performance variable such as force, torque, vibration, or temperature), and converting the predicted physical values at a future time to a conditional performance reliability, according to the given critical performance value $y_{C L}$ for the measure $y(t)$. Kolarik $(1988,1995)$ developed the concept and mathematics for performance conditional reliability based on a continuous scale of the physical performance of a system, subsystem, or component, which serves as the basis for the probabilistic output for any given failure mode. On this basis, given the definition of failure in terms of performance characteristics, the performance reliability is defined as the conditional probability that the performance measure $y(t+\Delta t)$ is less (or greater) than a performance critical limit $y_{C L}$ (which represents an appropriate definition of failure in terms of physical performance, $y(t), t=0,1,2 \ldots)$, given environmental conditions of operation, for a specified time period or usage $\Delta t$. Defining $T$ as a continuous random variable for the system operation failure time; let $F(t)$ denote the cumulative probability function of system failure for the performance $y(t)$. The system performance reliability, $R(t)$, can also be given by $R(t)=1-F(t)$. Then the conditional performance reliability can be evaluated as ,

$$
\begin{aligned}
& R((t+\Delta t) \mid t)=P\{T>(t+\Delta t) \mid T>t\} \\
& \quad=[1-F(t+\Delta t)] /[1-F(t)] \\
& \quad=R(t+\Delta t) / R(t)
\end{aligned}
$$

The conditional performance reliability $R((t+\Delta t) \mid t)$ represents the probability that the system will survive up to time or usage $t+\Delta t$, given the condition that the system performance has not failed up to time $t$. This concept and definition apply to all the three basic possibilities existing in applications: lower is better, a performance measure with an upper critical limit; higher is better, a performance measure with a lower critical limit; and nominal is best, a performance measure with a two sided critical limit. Figure 1 depicts the concept of performance reliability and a performance measure with its critical limit.

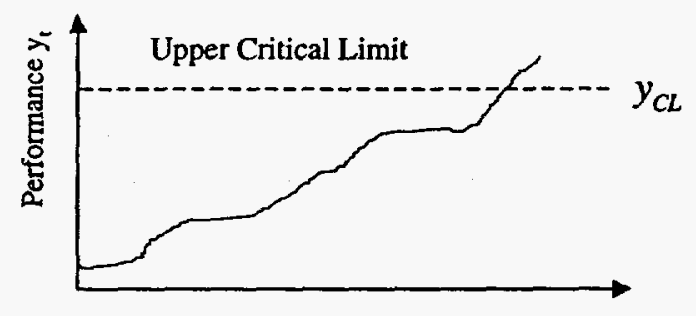

Time/Usage $t$

(a) Lower is better in physical performance.

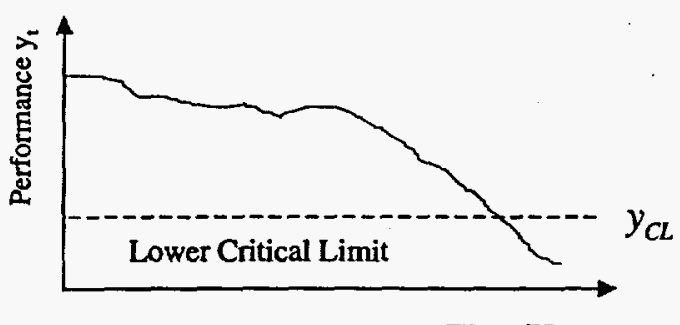

Time/Usage $t$

(b) Higher is better in physical performance.

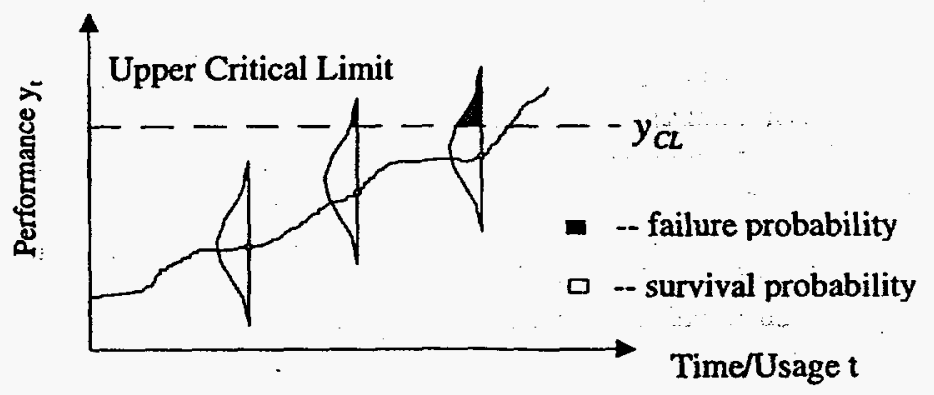

(c) Physical performance measure and performance reliability concept.

Figure 1. Performance measure and performance reliability. 
The shaded area of the individual performance distribution at time $t$ in Figure 1 (c) represents the probability of failure at time or usage $T \leq t$.

\section{TIME SERIES MODELING OF PERFORMANCE MEASURES}

The physical performance $y(t)$ is sampled across time. It can be modeled as a time series, a realization or sample function from the performance stochastic process $Y(\omega, t)$, where $\omega$ belongs to a sample space and $t$ belongs to an index set. In time series analysis, $y(t)$ is related to its past values and driven by a white noise process. A full model, the general AutoRegressive Integrated Moving Average model, $\operatorname{ARIMA}(p, d, q)$, is typically used to model a general discrete series in time,

$$
\phi(B)(1-B){ }^{d} y_{t}=\theta(B) a_{t}
$$

where

$$
\begin{aligned}
& \phi(B)=\left(1-\phi_{1} B-\ldots-\phi_{p} B^{p}\right) \\
& \theta(B)=\left(1-\theta_{1} B-\ldots-\theta_{q} B^{q}\right) \\
& B \text { is the back-shift operator } \\
& p, q \geq 0 \text { and } d>0
\end{aligned}
$$

$a_{t}$ is a zero mean white noise process with

variance $\sigma_{a}^{2}$.

In this model $p$ represents the order of the AR part, while $q$ represents the order of the MA part. If either $p$ or $q$ is zero, then the result is a subset, ARI (AutoRegressive Integrated) model or IMA (Integrated Moving Average) model, respectively. The integrated part in the model actually performs a differencing operation on the original series and the differenced series is stationary. Usually, in time series modeling the final model is chosen through the processes of model identification, model estimation and model validation.

For model structure fit, to the data set, the model order is determined from the Akaike information criterion (AIC):

$$
\operatorname{AIC}\left(N_{p}\right)=N \ln \left(\sigma_{a}^{2}\right)+2 N_{p}
$$

where $N$ is the number of data points

$\sigma_{a}^{2}$ is the residual variance

$N_{p}$ is the number of parameters in the model
Here, $N_{p}$ is $p+q$ for the ARMA model. The optimal order of the model is chosen by the value of $N_{p}$, which is a function of $p$ and $q$, so that $A I C\left(N_{p}\right)$ is a minimum (Wei, 1990).

For model validation, the model residuals are checked to see if the residuals of the model follow a white noise process. The forecasts based on a selected model can be obtained by setting $t=n+l$ in the general expression of the model,

$y_{t}=\phi_{1} y_{t-1}+\ldots+\phi_{p+d} y_{t-p-d}+a_{t}-\theta_{1} a_{t-1}-\ldots-\theta_{q} a_{t-q}$

for an $l$ - step ahead value, $y_{n+1}$, at time $n$. Equation (5) can be obtained from equation (3). In forecasting, for $t \leq n, y$, will be a known observation and the error term $a_{1}$ is replaced by its estimate, the residual $\hat{a}_{t}$; for $t>n, y_{t}$ is replaced by its forecast counterpart at time $n$ and the unknown $a_{t}$ is set to zero (Newbold \& Bos, 1994). The underlying variations in performance around the forecast values are calculated and converted to the reliability prediction. Here, $a_{t}$ is assumed to follow a normal distribution.

\section{DATA, MODELING AND RESULTS}

In general, the time series approach is applicable to all engineering systems for real-time assessment of system survival, provided performance variables and their critical limit values are properly defined. In this paper, a drilling operation is illustrated as an example for survival assessment of drill bit performance. The thrust force of a drill bit in operation is chosen as a physical performance measure for the performance failure of the drill with respect to an excessive wear failure mode (Thangaraj and Wright, 1988). In the experiment, the thrust cutting force (in pounds) was sensed with a Kistler 9271A force and torque dynamometer. The average thrust force collected and extended from a drill bit in its 45-hole drilling operation is displayed in Figure 2. The average thrust force is defined as the average value of the thrust cutting force on the drill in each hole drilling operation from full-cut start to the full-cut end. The average thrust cutting force values in the $\mathbf{4 5}$ holes represent a time series $y(t)$. It is clear that the thrust cutting force trend increases with the hole numbers (as we would expect due to tool edge wear). Detailed information about the experimental data set is available, see Kim 1991. 


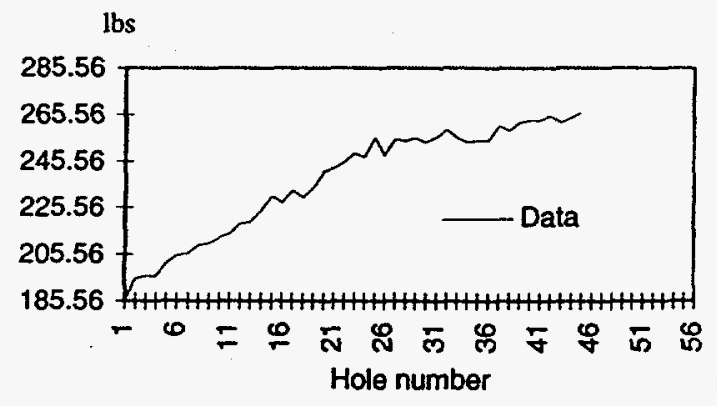

Figure 2. Thrust force data from a drill bit operation.

In order to model the performance measure and assess reliability, a first difference of series is made and then the differencing residuals are fit to an ARMA model. The model structure and model order is determined by the AIC value and white noise test for model residuals. Table 1 shows the results in model identification, where Wntest $p$ represents the p-value of Bartlett's white noise test and Rvar is the residual variance of the model (Newton, 1988).

Table 1. Model selection for the data set.

\begin{tabular}{|l|l|l|r|}
\hline Model & AIC & Wntest p & \multicolumn{1}{l|}{ Rvar } \\
\hline $\operatorname{ARMA}(1,1)$ & 107.884 & 0.9792 & 10.1304 \\
\hline $\operatorname{ARMA}(2,1)$ & 108.410 & 0.9271 & 9.7944 \\
\hline $\operatorname{ARMA}((2), 1)$ & 107.110 & 0.9853 & 9.9537 \\
\hline $\operatorname{ARMA}(2,2)$ & 110.425 & 0.9904 & 9.7980 \\
\hline $\operatorname{ARMA}(3,2)$ & 115.643 & 0.9397 & 10.5436 \\
\hline
\end{tabular}

According to the AIC value and the white noise test for model residuals, model ARMA((2), 1) with two parameters is chosen as a parsimonious model for the data set. Including the first difference for the data set, the full model can be estimated as $\operatorname{ARIMA}((2), 1,1)$,

$$
\left(1+0.368 B^{2}\right)(1-B) y_{t}=(1+0.308 B) a_{t}
$$

where $B$ is the back-shift operator, $a_{t}$ is a zero mean white noise process with the variance of 9.9537 . The parameters are estimated from the estimate macro in TIMESLAB (Newton, 1988).

Once the final model is determined, the forecasts are made based on this model. In general, there are different types of possible forecasts. For the purpose of reliability assessment, a $l$ step ahead forecast is used. Here data values available up to time $t$ are used to forecast the value at time $t+l$ based on the model. In this experiment, 42 data points are used for modeling and $l$ is set to 1 . In total another 18 point forecasts are calculated up to index 60 . The forecasts are obtained from the forecast macro in TIMESLAB. Figure 3 shows the original data and the model forecasts.

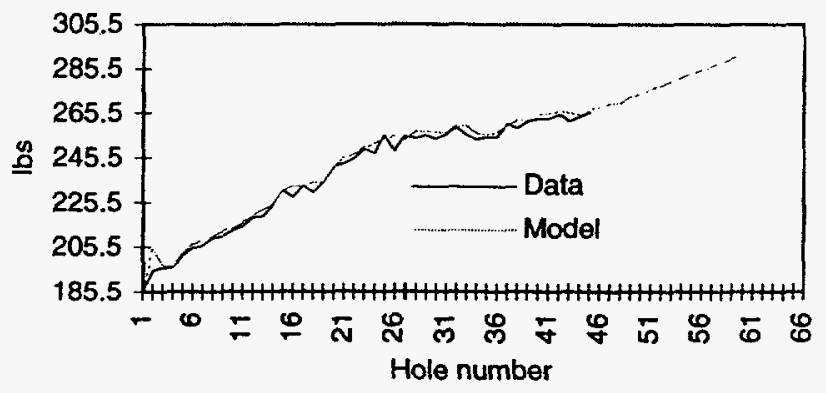

Figure 3. Model and prediction for the data set.

In the forecasting of this application, the ARTMA model is used to establish the performance trend, as shown in Figure 3. This trend dictates the location for the predicted probability distribution. Let $S$ denote the probability of success of the operation at hole $t$ (where the forecast value of performance $\hat{y}(t)$ is less than or equal to the critical limit value $\left.y_{C L}\right)$. Assuming the predicted performance distribution is normally distributed and has variance $\sigma^{2}$, then $S$ can be evaluated as

$$
\begin{aligned}
S_{t} & =P(\text { success for hole } t) \\
& =1-\int_{y}^{\infty} g\left(y_{t}\right) d y_{t}
\end{aligned}
$$

where $g\left(y_{t}\right) \sim N\left(\hat{y}(t), \sigma^{2}\right)$

$\hat{y}(t)$ is model forecast value at hole $t$

$y_{C L}$ is the given critical limit value

$t$ is a hole index number; $t=42,43, \ldots$

Table 2 (in column 3 ) shows the estimates of individual hole success probabilities in forecasting, from the $42^{\text {nd }}$ hole, based on the model. The conditional performance reliability for a series of holes, given survival to the $42^{\text {nd }}$ hole, is calculated and shown in the fourth column of Table 2. 
Table 2. Performance predictions and conditional reliability estimates.

\begin{tabular}{|l|l|l|l|}
\hline Index $n$ & $\begin{array}{l}\text { Hole } \\
\text { number } \\
,\end{array}$ & $\begin{array}{l}\text { Individual } \\
\text { hole } \\
\text { predictions } \\
S_{1}\end{array}$ & $\begin{array}{l}\text { Conditional } \\
\text { reliability } \\
\text { survival to } \\
42^{\text {nd }} \text { hole }\end{array}$ \\
\hline 0 & 42 & 1.000 & 1.000 \\
\hline 1 & 43 & 0.998 & 0.998 \\
\hline 2 & 44 & 0.996 & 0.994 \\
\hline 3 & 45 & 0.995 & 0.989 \\
\hline 4 & 46 & 0.990 & 0.979 \\
\hline 5 & 47 & 0.983 & 0.962 \\
\hline 6 & 48 & 0.980 & 0.943 \\
\hline 7 & 49 & 0.941 & 0.887 \\
\hline 8 & 50 & 0.893 & 0.792 \\
\hline 9 & 51 & 0.739 & 0.628 \\
\hline 10 & 52 & 0.614 & 0.385 \\
\hline 11 & 53 & 0.341 & 0.132 \\
\hline 12 & 54 & 0.184 & 0.024 \\
\hline 13 & 55 & 0.060 & 0.002 \\
\hline 14 & 56 & 0.020 & 0.000 \\
\hline 15 & 57 & 0.005 & 0.000 \\
\hline 16 & 58 & 0.001 & 0.000 \\
\hline 17 & 59 & 0.000 & 0.000 \\
\hline 18 & 60 & 0.000 & 0.000 \\
\hline
\end{tabular}

The individual performance reliability shown in Table 2 is equivalent to a mission reliability where, in this example, the mission constitutes the probability of successfully drilling one more hole, given that the drill has survived through the previous holes. Mission definition depends on applications and affects the $l$ value of forecasting in time series modeling. The conditional performance reliability curve, given the survival to the $42^{\text {nd }}$ hole, is calculated from the individual performance reliability values from each observation. For example, the performance reliability that a particular drill bit would survive $n$ holes is simply the product of the individual performance reliability values of surviving each of these $n$ holes (basically the product of $n$ individual performance reliability values).

$$
R\left(t_{42+n^{1}}^{\mid t}\right)=\prod_{i=42}^{42+n} S_{i}
$$

In this application, $y(t)$ is thrust force in pounds and $y_{C L}$ is the critical limit value set to $275 \mathrm{lbs}$, regarding a drill failure mode of excessive wear. Figure 4 shows the predicted conditional reliability curve, given the survival to the $42^{\text {nd }}$ hole. For example, the conditional reliability of the $45^{\text {th }}$ hole operation is estimated as 0.989 .

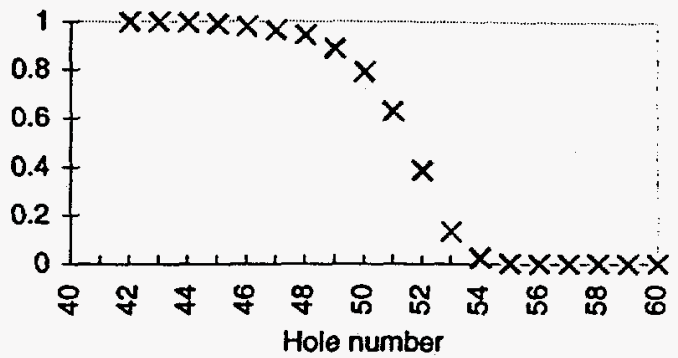

Figure 4. Conditional performance reliability curves, given the survival to $42^{\text {nd }}$ hole $\left(\mathrm{y}_{\mathrm{Cl}}=275 \mathrm{lbs}\right)$.

The curve reveals the estimates of conditional reliability for each drilling operation after it survived the $42^{\text {nd }}$ hole. For example, the conditional reliability for the $45^{\text {th }}$ hole is estimated as 0.989 , and 0.792 for the $50^{\text {th }}$ operation. This information is useful in system operations (e.g., in predictive maintenance).

\section{CONCLUSIONS}

Ideally, self-assessment of survival for a system, subsystem or component is implemented by assessing conditional performance reliability in real-time, which includes modeling and analysis of physical performance data. This paper describes a time series analysis approach to system self-assessment (prediction) of survival. In this time series approach, performance data are modeled in a time series. The performance forecasts developed are based on the model and are converted to system survival probabilities (reliability). The results presented in this paper constitute an example of time series modeling and survival assessment, regarding an excessive tool edge wear failure mode for a twist drill operation. In contrast to a standard regression model, a time series model, using single channel data is a suitable alternative for real-time performance prediction.

This paper features a single channel (drill thrust) input, with a single cutting edge wear failure mode related reliability output. The time series modeling approach is compatible with, and can be extended to multivariate performance.

\section{ACKNOWLEDGMENT}

The research work described in this paper is partially funded through The Texas Advanced Research Program and The ANRCP, Robotics, Automation, and TeleOperations Program. 


\section{REFERENCES}

1. Chinnam, R. B., Kolarik, W. J. and Manne, C. V., (1996), "Performance Reliability Prediction Using the Validity Index Neural Network," International Journal of Modelling and Simulation, Vol. 16, No. 4, pp. 210217.

2. Kim, Y.S. and Kolarik, W. J., (1992), "Real-Time Conditional Reliability Prediction From On-Line Tool Performance Data," International Journal of Production Research, vol. 30, no. 8, pp. 1831-1844.

3. Kim, Yon-Soo, (1991), "Performance and Tool Reliability for Metallic Composites," Ph.D. dissertation, Texas Tech University.

4. Kolarik, W. J., (1988), "Performance Reliability," working paper, Industrial Engineering Dept., Texas Tech University.

5. Kolarik, W. J., (1995), Creating Quality: Concepts, Systems, Strategies, and Tools, New York, NY: McGraw-Hill.

6. Newbold \& Bos, (1994), Introductory Business \& Economic Forecasting, South-Western Publishing Co.

7. Newton, H. Joseph, (1988), TIMESLAB: A Time Series Analysis Laboratory, Wadsworth, Inc..

8. Thangaraj, A. and Wright, P.K., (1988), "Drill wear sensing and failure prediction for untended machining,"
Robotics \& Computer-Integrated Manufacturing, Vol.4, No. 3/4, pp. 429-453.

9. Wei, Willian W. S., (1990), Time Series Analysis: Univariate and Multivariate Methods, Addison-Wesley Publishing Company.

\section{BIOGRAPHICAL SKETCHES}

HUITIAN LU is a graduate student in the Industrial Engineering Department of Texas Tech University. He has over 4 years of work experience in quality control with Automated Defect Detection and Classification (ADDC) in semiconductor wafer manufacturing. His current research interests are in reliability modeling, survival assessment, and quality control. $\mathrm{H}$. Lu is an active member of SME.

WILLIAM KOLARIK is a Professor in the Department of Industrial Engineering at Texas Tech University. He currently serves as the Director of the Center for Applied Research in Automation and Robotics. He has approximately 12 years experience in teaching and research in the fields of quality and reliability. He has published over 50 technical publications and papers and a major textbook (Creating Quality, McGraw-Hill, 900+ pages) on both quantitative and qualitative aspects of quality and reliability. 